\title{
OPTIMIZATION APPROACHES IN OPTIMAL INTEGRATED CONTROL, COMPUTATION AND COMMUNICATION SYSTEMS
}

\author{
M.M. Share Pasand \\ Department of Electrical and Electronics Engineering, Faculty of Electrical, Mechanical \\ and Civil Engineering, Standard Research Institute, Iran
}

\begin{abstract}
This paper studies the existing approaches in optimal integrated control, computation and communication problems. It concentrates on joint optimization problems aimed at finding communication/computation policy and control signal. Different aspects including computational complexity, convexity, proposed methods to find optimum and other issues related to control performance are studied and compared for different approaches.
\end{abstract}

\section{KEYWORDS}

Integrated Control and Communication, Networked Control Systems, Constrained Optimization.

\section{INTRODUCTION}

Data communication networks are utilized in most of modern control systems, as a mean to transmit measurement/control data between plant(s) and controller(s). Control systems utilizing communication networks are first referred to as integrated control and communication systems (ICCS). [1-3] The main characteristic of an ICCS is that they require data transmission for desirable performance using limited communication resources. An ICCS could be a distributed control system including distributed sensors/actuators controlled by a single controller or a group of controllers which is known as a Networked Control System (NCS). Also an embedded single loop control system with a limited communication channel or computation capacity which requires additional measures to be considered, is an ICCS. [7] surveys issues arises in an ICCS when integrating control and communication. Control methods used in this environment are studied and surveyed in [8-11]. A survey of network-induced problems which affect control systems is reported in [12].

Another group of control systems which are similar to ICCS in several aspects are integrated control and computation systems. These systems control a group of plants using limited computational (rather than communication) resources. In these applications, a single processor is shared among several control tasks or have to multi-task between a single control application and some other tasks. Adaptive and on-line optimal control algorithms as well as estimation problems require significant computational overhead. In these applications, processor time should be scheduled in an efficient manner to yield proper control performance. Examples could be found in [4-6].

Therefore, control systems are emerging into control, computation and communication systems in which an efficient control algorithm should account for communication and computation resources. In a more general framework, one may refer to theses systems as Integrated Control, Computation and Communication Systems (ICCCS). Figure 1, depicts the scope of ICCC problems. 


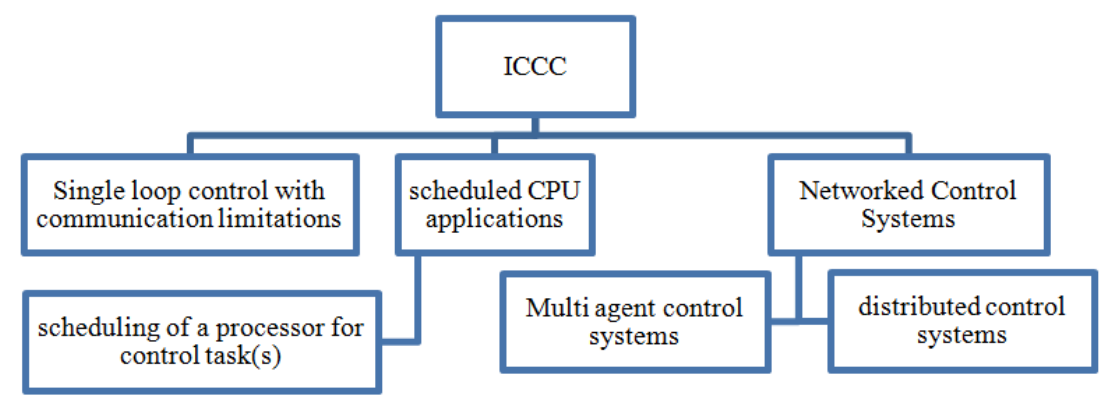

Figure 1: Scope of ICCC Problems

The objective of an ICCC problem is to find a control algorithm alongside with management policies for computational and communication resources to yield best possible performance. There are different approaches in tackling the ICCC problem. In several applications, both communication and computation constraints lead to or could be assumed to result in similar problems (i.e. delays, outdated information, etc.) therefore most of previous works concentrated on only one aspect. In networked control systems, communication resources are more important than computational ones and therefore control and communication are considered in the problem and computational issues are neglected [1,2,13-17], While [4-6] and references therein consider only computational limitations assuming that communication channel is perfect or not present.

\section{ICCC Problem}

The aim of an ICCCS is to compute a control input, a communication policy (which consists of bit rate selection [5] or medium access scheduling [13,14,18-21] in contention-free networks and/or methods to confine bandwidth usage [22,23] in contention-based networks [17]), which (in contention-free cases) results in a communication sequence determining which node is to be granted medium access and/or a computation policy which schedules different computational tasks to be performed. This is an integrated problem in nature as it includes interacting objectives. Typical objectives include quadratic forms of state and control inputs as $[5,13]$ and network induced error of output [45] or state [29]. Communication and computation limitations may be implemented as constraints of optimization problem as in [13], incorporated into state space model itself [17] or directly added to the quadratic objective as communication or computation costs [51].

\subsection{Approaches to ICCC Problem}

There are three approaches to solve ICCC problems. These approaches may be regarded as either modelling or solving approaches.

The first approach is to solve control problem with regard to communication system situation referred to as communication aware control [24] and could be found for instance in [25-28]. Communication aware control methods require an estimation of network traffic or delay to amend control policy respectively. Communication aware approach is best fitted to applications utilizing contention-based networks. This is due to the fact that a contention-based network may not provide constant available bandwidth and may suffer from packet losses or transmission delays as a result of varying workload [30,31], therefore it has a time-varying status which is required to be considered when control input is determined. In this approach, a control algorithm may be required to reduce its communication in order to save bandwidth. Examples include interrupt based control [49], event driven control [50] and joint LQG control of [51] which adds a communication cost to the traditional quadratic cost function. 
The second approach is to find and pre-compute an appropriate communication policy based on the plant dynamics and/or other measures, in offline manner. This method may be regarded as control aware communication [24] in contrast to the first approach. In this approach, communication sequences are chosen based on control objectives. Then control inputs are computed online and applied via the communication sequence. This approach is appropriate for contention-free networks with the ability to pre-determine which nodes are to be granted medium access. Examples include state aware feedback scheduling [24] used in [18-22,29]. The offline communication sequence should be chosen in such a way that provides a minimum level of appropriateness. For example communication sequences which preserve controllability or observability of the NCS as discussed in [17,29,32-34].

The third approach is to integrate both problems to find optimal/sub-optimal solutions in online or semi-online manner as in $[4,5,13,16,20]$. This approach may be utilized when abundant computational resources are present and communication constraints are restrictive, affecting control performance and stability in an adverse manner. In this method, computation/communication and control policies are determined via solving a joint optimization problem.

Communication decisions may be made online or offline. Online decisions on communication policy includes selection of communication sequences, selection of sampling intervals, determination of quantization thresholds, etc. On the other hand, control input computation is always online due to the dynamic nature of feedback control. Table 1, illustrates the aforementioned approaches.

Table 1: Approaches in ICCC

\begin{tabular}{|l|l|c|c|}
\hline \multicolumn{1}{|c|}{ Approach } & Method of incorporating communication & Examples & Type of network \\
\hline $\begin{array}{l}\text { 1.(Communication } \\
\text { aware control) }\end{array}$ & $\begin{array}{l}\text { Online Estimation of QOS (transmission } \\
\text { delays, packet losses, etc.) }\end{array}$ & {$[25-28]$} & Contention-based \\
\hline $\begin{array}{l}\text { 2.(Control aware } \\
\text { Communication) }\end{array}$ & $\begin{array}{l}\text { Offline computation of communication } \\
\text { sequence }\end{array}$ & {$[17,29,32-34]$} & Contention free \\
\hline $\begin{array}{l}\text { 3.(Integrated } \\
\text { control and } \\
\text { scheduling) }\end{array}$ & $\begin{array}{l}\text { Online, simultaneous computation of } \\
\text { control input, state estimations and } \\
\text { communication/computation policies. }\end{array}$ & {$[4,5,13,16,20]$} & Contention free \\
\hline
\end{tabular}

\subsection{Computational Complexity}

Although there are simple methods in the literature like [35], the first approach usually requires the minimization of predicted cost functions [25-28]. As plant state vector is not known as a priori, optimizations could not be performed offline and therefore this approach is not appropriate for systems with fast responses and/or low computational power. To the best of the authors knowledge, it is the only existing approach in contention-based paradigm.

The second approach is appropriate for most applications in contention-free paradigm as it precomputes communication sequences to fulfil communication constraints. This method may not be optimal with regard to performance or bandwidth usage, however it is simple and computationally efficient.

The third approach for solving ICCC problem usually consists of optimization problems with complexity of high orders. Usually numerical methods should be used to solve these problems. [13] could be referred for an experimental and simulation example. 


\section{CoMparison OF EXISTING Methods}

In this section, existing approaches are studied and compared with regard to computation complexity, type of optimization problems and control performance considerations.

\subsection{Optimal Integrated Control and Scheduling}

In [13], a moving horizon quadratic cost function is introduced to represent control performance and stability concerns. Scheduling is incorporated in the model as a set of constraints forming a hybrid model predictive control problem (HPC) consisting of quadratic cost function of continuous time variables (control signal vectors) and constraints of continuous and integer variables.

\subsubsection{Optimization Problem}

The optimization problem could be stated as follows [13]:

$$
\left\{\begin{array}{l}
\min x^{T}(N) S x(N)+\sum_{k=0}^{N-1}\left[\begin{array}{l}
x(k) \\
u(k)
\end{array}\right]^{T} Q\left[\begin{array}{l}
x(k) \\
u(k)
\end{array}\right] \\
\text { s.t: }\left\{\begin{array}{l}
x(k+1)=A x(k)+B u(k) \\
\delta_{i}(k) \in\{0,1\} \\
\sum_{i=1}^{m} \delta_{i}(k) \leq b \\
u_{i}(k)-u_{i}(k-1)=\delta_{i}(k) u_{i}(k)-\delta_{i}(k) u_{i}(k-1) \\
x(0)=x_{0}
\end{array}\right.
\end{array}\right.
$$

This model leads to a Mixed Integer Quadratic Programming (MIQP) with a convex cost function and nonlinear (multiplicative) constraints. Note that by relaxing integer constraint $\delta_{i}(k) \in\{0,1\}$ into $0 \leq \delta_{i}(k) \leq 1$, (namely QP relaxation) incorporating all variable in a time frame of $N$, and defining a new variable vector as $z(k)=\left[\begin{array}{ll}u^{T}(k) & \delta^{T}(k)\end{array}\right]^{T}$, this problem could be reformulated to form a standard Quadratically Constrained Quadratic Programming (QCQP) problem. The third constraint is indeed a linear inequality and the fourth could be restated as a quadratic equality constraint. After finding the optimum variable, scheduling sequence could be derived as the following, leading to a sub-optimal solution.

$$
\hat{\delta}_{i}(k)=\left\{\begin{array}{cc}
0 & \delta_{i}(k) \leq 0.5 \\
1 & \delta_{i}(k)>0.5
\end{array}\right.
$$

\subsubsection{Computational Complexity}

Optimization problem (1), should be solved by extending variable vectors for all times within the prediction horizon $(N)$. This enlarges the problem as $N \times 2 m$ variables (all $u_{i}(k), \delta_{i}(k), k=$ $0, \ldots N)$. For a fixed set of scheduling variables, the problem is a QP which could be solved using simple analytical and numerical methods. Therefore, (1) is of combinatorial complexity as it include solving a QP of size $N \times m$ (a predictive optimization with $m$ variables and a horizon of $N$ ) for all possible combinations of $\delta_{i}(k) s$ considering (1.3). Number of possible combinations for scheduling variables could be computed from figure 1 : 


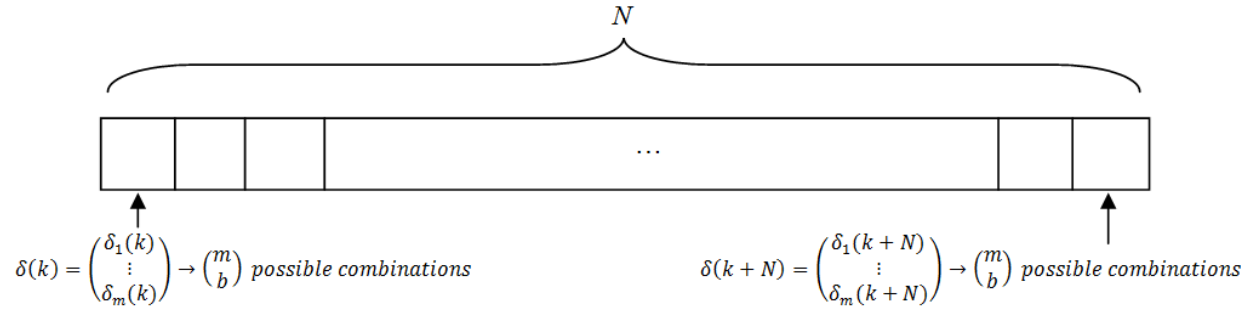

Figure 2. Communication Sequence

which is $\left(\begin{array}{c}m \\ b\end{array}\right)^{N}$ possible combinations. Assuming linear complexity for QP problems, the computation time for a complete search over all possible communication sequences will be:

$$
\left(\begin{array}{c}
m \\
b
\end{array}\right)^{N} \times O_{Q P}(2 N m)
$$

$O_{Q P}(l)$ represents computational complexity of a constrained quadratic programming problem with dimension $l$. Increasing the prediction horizon will enlarge dimensions in an exponential manner. Unfortunately increasing the horizon is the most common way in predictive approaches to guarantee stability or enhance stability robustness [36]. In fact formal proofs for stability of MPCs is only proposed for infinite horizon cases. [37,13]. For a simple case of two inputs and a unity bandwidth network, a horizon of 10 , results in 1024 combinations which is barely practical to be computed within each sample time.

To reduce computational overhead, one may restrict communication sequences to a few proper choices. For this purpose a simplified method namely OPP is proposed by [13]. OPP restricts the search space of communication sequences to $m$. In other words, a constant periodic communication sequence is formed and the algorithm only chooses the starting point. Therefore number of combinations reduce from $\left(\begin{array}{c}m \\ b\end{array}\right)^{N}$ to $m$.

\subsubsection{Existing Methods for Solution}

MIQP problems are usually solved via one of the common approaches known as Cutting plane methods, decomposition methods, logic-based methods and branch and bound methods [38-40]. Branch and bound methods are known to be the most efficient approaches for convex MIQP[41] and used in [13]. Branch and Bound methods, usually use QP relaxation as mentioned in section A.1. In this method, ordinary constrained QP problems are derived via relaxing the integer constraints to interval constraints. The created ordinary QP problems are solved in the nodes. As the method makes progress down in the tree, fixed integer variables are eliminated from the problem. This means that the number of optimization variables in the relaxed sub-problems decreases by one for each level passed on the way down the tree. [40] For non-convex MIQP problems, spatial branch and bound methods could be used. MATLAB, CPLEX and YALMIP are of the existing software to solve this type of problem. [42]

\subsubsection{Other Issues}

Note that (1) should be performed on-line during each period, this makes the algorithm agile, capable of yielding high performance in presence of disturbances and adaptability with respect to model changes. However implementing (1) is computationally impractical. There is no guarantee for stability unless for infinite horizon[13]. OPP is simpler and still better than static scheduling [13]. Also note that (1) greedily demands for bandwidth usage as there is no constraint on the frequency of input updates. 


\subsection{Optimal Offline Periodic Scheduling}

In [29], the problem of scheduling a set of actuators belonging to a set of processes is investigated. The aim is to find optimal LQ gain for each process to implement a state feedback controller as well as finding optimal communication logics. This is a special case of the general problem in which sensors and actuators are subject to communication constraints and plants are not necessarily disjoint.

\subsubsection{Optimization Problem}

A quadratic cost function is derived via sampling of a continuous time cost function. The optimization problem could be state as follows:

$$
\begin{gathered}
\min \sum_{i=1}^{m} w_{i} f_{i}(s) \\
\text { s.t. }\left\{\begin{array}{c}
s \in S_{p} \\
m \leq p \leq p_{\max }
\end{array}\right.
\end{gathered}
$$

$S_{p}$ is the set of possible $p$-periodic communication sequences for sensors. The cost function is a weighted sum of maximum deviation of each process quadratic cost function from the optimal cost function derived via solving a Ricatti equation. i.e.:

$$
f_{i}(s) \triangleq \max _{\|x\| \neq 0} \frac{x^{T} \sum_{k=1}^{p} \frac{1}{p} P_{k}(s) x}{x^{T} P^{c} x}-1
$$

$P^{c}$ is the positive definite solution to continuous time Ricatti equation of the original continuous time system and $P_{k}(s)$ is the solution to Ricatti equation associated with the following LQ problem.

$$
\left\{\begin{array}{c}
\min x^{T}(N) S x(N)+\sum_{k=0}^{N-1}\left[\begin{array}{l}
x(k) \\
u(k)
\end{array}\right]^{T} Q\left[\begin{array}{l}
x(k) \\
u(k)
\end{array}\right] \\
\text { s.t: }\left\{\begin{array}{c}
x(k+1)=A x(k)+B u(k) \\
x(0)=x_{0}
\end{array}\right.
\end{array}\right.
$$

This problem consists of two optimizations. (2) is a combinatorial optimization problem and could be restated as a Binary Linear Programming by defining the elements of sequence $S$ as optimization variables, if all possible sequences are admitted. (4) is a quadratic non-constrained problem having an analytical solution based on Ricatti equation. [17] utilized Genetic Algorithms and Particle swarm optimization to compute the optimal communication sequence. Then it compared its methods with that of [29] via simulations.

\subsubsection{Computational Complexity}

Due to combinatorial nature of (2), it becomes exponentially complicated when the period of communication sequence increases. (2) requires searching through a set of $\sum_{j=m}^{p_{\max }} 2^{j}$ each time solving a LQ problem of dimensions equal to original system dimensions. Computation complexity will be: 
International Journal of Control Theory and Computer Modeling (IJCTCM) Vol.6, No.4, October 2016

$$
O_{L Q}(n) \sum_{j=m}^{p_{\max }} 2^{j}
$$

$O_{L Q}(n)$ is the computational complexity of a Linear Quadratic Control problem which is usually performed by solving a Ricatti equation. Note that if $p_{\max }$ is not known or fixed as a priori, computations may grow until a feasible sequence is achieved. [29] (3) and (4) could be performed off-line or semi-online to result in periodic communication sequences of period $p$. Therefore computational complexity is not a concern as it is in [13].

\subsubsection{Existing Methods for Solution}

[17] used GA and PSO to search for the optimal communication sequence. [29] uses a simpler but less accurate method. In [17] the results are compared.

\subsubsection{Other Issues}

(2) is only appropriate for sensor scheduling and only applies to a situation in which a number of plants are scheduled via a shared bus. When the plants have interaction (i.e. several output channels of a single MIMO process are to be scheduled) or when the network bandwidth is more than unity, the formulation should be extended.

\subsection{Optimal Sample Period Assignment}

[5] proposes a method for on-line sample period assignment in a feedback scheduling time window to control several plants connected to a shared processor. Sample time assignment is in fact an inherent way to schedule network access or assign bandwidth proportions to nodes. Therefore this situation is similar to that of case 3.2.However [5] assumes that both sensors and actuators of each plant have the same sampling frequency.

\subsubsection{Optimization Problem}

The cost function is the sum of individual quadratic cost functions for each plant. It could also be a weighted sum of all cost functions. There are constraints regarding schedulability and minimum sampling intervals. It is desired to find sample intervals for each plant in order to minimize the overall cost function.

$$
\begin{gathered}
\min \sum_{i=1}^{m} J_{i} \\
\text { s.t. }\left\{\begin{array}{l}
h_{1} \ldots h_{n} \\
\sum_{i=1}^{m} \frac{E_{i}}{h_{i}} \leq U_{s p}-U_{f s} \\
h_{\text {min }} \leq h_{i} \leq h_{\text {max }} \quad i=1, \ldots m
\end{array}\right.
\end{gathered}
$$

$U_{s p}$ is the utilization set point of processor and $U_{f s}$ is the utilization occupied by overhead computations. $E_{i}$ is the execution time of control task associated with $i^{\text {th }}$ plant.[5].

The problem is convex with regard to $\frac{1}{h_{i}}$ if (5.2) is relaxed. Therefore one may relax (5.2) and solve (5) as a QCQP. After solving the problem, if the resultant $h_{i}$ does not fulfill (5.2), it should be replaced by $h_{\min } / h_{\max }$. Note that (5.2) could be extended to $n$ constraints each of which associated with each plant. (i.e. $h_{\text {min }}^{i} \leq h_{i} \leq h_{\text {max }}^{i}$ ) 


\subsubsection{Computational Complexity}

(5) should be solved every $T^{f b s}$ step which is similar to prediction horizon $N$ in [13]. $\mathrm{N}$ contrast to methods investigated in sections $\mathrm{A}$ and $\mathrm{B}$, this problem is computationally simple and consists of a near-standard optimization problem. The problem is not of a combinatorial complexity and increasing $T^{f b s}$ does not adversely affect the complexity.

[5] proposed to form a pre-computed table (table 1, [5]) of cost functions and sample periods to save processor utilization. The table is computed off-line and is used as a look up, during on-line optimization. Linear search is applied to compute the look up table which seems sufficient though other search methods could be utilized. The optimization problem is of order $O(\mathrm{mH})$ (linear order) in which $m$ is the number of plants (control tasks) and $H$ is the number of possible sampling periods for each plants.

\subsubsection{Existing Methods for Solution}

Solving (5) does not need complicated methods and is simple enough to be implemented on embedded systems and simple firmware.

\subsubsection{Other Issues}

Although (5) is simple, it can't be directly implemented to tackle problems as for section A or 3.2.[5] aims to find sampling intervals for control tasks which is scheduling of the processor time while A and B are seeking to schedule network access. Network access schedules could not be pre-emptive. Also information of states (and even output) are not always available.

A common drawback of methods introduced in sections B and C is that they consider a number of disjoint plants and it is assumed that network bandwidth is enough to almost instantly transmit each plant information to/from controller. This contradicts with many realistic situations in which many interacting input/output channels are to be scheduled to have access to network. Also it is shown in previous works (e.g. [13]) that the insertion of a shared network results in interactions between disjoint plants.

Another limitation of (5) is that it relies on the concept of sampling interval which assumes that all sensors and actuators of a specific plant are sampled via the same sample time. In practice it may be desired for an output channel to be sampled more frequently than the other.

\subsection{Integrated Control and Scheduling Based on Network Induced Error}

[44] considers the relative network induced error of state as the cost function, which is similar to the idea of Maximum Error First (MLF) of [45] but uses relative state error rather than absolute output error.

\subsubsection{Optimization Problem}

The optimization problem is as follows:

$$
\begin{gathered}
\min \sum_{i=1}^{m} \frac{\left|x_{i}(k)-\hat{x}_{i}(k)\right|}{\left|\hat{x}_{i}(k)\right|} \\
\text { s.t. }\left\{\begin{array}{c}
\sum_{i=1}^{m} \frac{E_{i}}{h_{i}}+\frac{B_{i}}{h_{i}} \leq m\left(2^{1 / m}-1\right) \\
h_{i} \leq h_{\max }-B_{i} \quad i=1, \ldots m
\end{array}\right.
\end{gathered}
$$


$\hat{x}_{i}(k)$ is the updated state vector existing within the controller while $x_{i}(k)$ is the actual state vector for $i^{\text {th }}$ plant. $B_{i}$ is the worst case time during which a signal may be blocked from being transmitted. (6.1) is the schedulability criteria for non-preemptive rate monotonic scheduling. This problem is non-convex as $\hat{x}_{i}(k)$ depends on sample time and is incorporated in a nonconvex manner into (6). (6.1) is a convex constraint with regard to $\frac{1}{h_{i}}$ and (6.2) is convex in $h_{i}$. (This issue could be resolved using the technique discussed in section 3.3.)

\subsubsection{Computational Complexity}

The computational complexity of this problem is similar to that of section 3.3. Especially when a look-up table is pre-computed, the problem becomes simpler. This problem is semi-online similar to that of section 3.3 .

\section{D.3. Existing methods for solution}

[44] used Genetic Algorithm to solve (6). Methods similar to those discussed in section C could also be utilized.

\subsubsection{Other Issues}

From a control point of view, (6) is not the best performance index to be minimized. A small relative error guarantees that communication medium is providing fresh data but it does not guarantee that control performance is desirable. In fact, (6) relies on the inherent assumption that paying more attention to the plant with enlarged network induced error will cause better performance. This depends on the effectiveness of control algorithm implemented in the controller. Approaches similar to [44] are frequently discussed in the literature. Examples include [45]-[48].

\section{Conclusions}

Integrated approaches for communication and control usually lead to combinatorial optimization problems and have to be simplified in order to be performed on-line. Among existing methods, LQR approach of [5] is the most promising as it is computationally simple however it does not account for shared network which is an important subject. The approach taken in [13] is based on realistic assumptions on network bandwidth limitations and interactions between different input/output channels , however it leads to complicated combinatorial optimization which takes significant time to be performed.

\section{REFERENCES}

[1] Halevi, Yoram, and Asok Ray. "Integrated communication and control systems: Part I-Analysis." Journal of Dynamic Systems, Measurement, and Control 110.4 (1988): 367-373.

[2] Ray, Asok, and Yoram Halevi. "Integrated communication and control systems: Part II-design considerations." Journal of Dynamic Systems, Measurement, and Control 110.4 (1988): 374-381.

[3] Luck, Rogelio, and Asok Ray. "Experimental verification of a delay compensation algorithm for integrated communication and control systems." International Journal of Control 59.6 (1994): 13571372.

[4] Cervin, Anton. Integrated control and real-time scheduling. Diss. Lund University, 2003.

[5] Cervin, Anton, et al. "Optimal online sampling period assignment: Theory and experiments." Control Systems Technology, IEEE Transactions on 19.4 (2011): 902-910.

[6] Castané, Rosa, et al. "Resource management for control tasks based on the transient dynamics of closed-loop systems." Real-Time Systems, 2006. 18th Euromicro Conference on. IEEE, 2006.

[7] Baillieul, John, and Panos J. Antsaklis. "Control and communication challenges in networked realtime systems." Proceedings of the IEEE 95.1 (2007): 9-28. 
[8] Tipsuwan, Yodyium, and Mo-Yuen Chow. "Control methodologies in networked control systems." Control engineering practice 11.10 (2003): 1099-1111.

[9] Hespanha, Joao P., Payam Naghshtabrizi, and Yonggang Xu. "A survey of recent results in networked control systems." PROCEEDINGS-IEEE 95.1 (2007): 138.

[10] Alur, R., et al. Handbook of networked and embedded control systems. Eds. Dimitrios HristuVarsakelis, and William S. Levine. Springer Science \& Business Media, 2007.

[11] Yang, Tai C. "Networked control system: a brief survey." IEE Proceedings-Control Theory and Applications 153.4 (2006): 403-412.

[12] Zhang, Lixian, Huijun Gao, and Okyay Kaynak. "Network-induced constraints in networked control systems-a survey." Industrial Informatics, IEEE Transactions on 9.1 (2013): 403-416.

[13] Gaid, ME Mongi Ben, Arben Cela, and Yskandar Hamam. "Optimal integrated control and scheduling of networked control systems with communication constraints: application to a car suspension system." Control Systems Technology, IEEE Transactions on 14.4 (2006): 776-787.

[14] Al-Areqi, Sanad, Daniel Gorges, and Steven Liu. "Event-Based Control and Scheduling Codesign: Stochastic and Robust Approaches." Automatic Control, IEEE Transactions on 60.5 (2015): 12911303.

[15] Garcia, Eloy, and Panos J. Antsaklis. "Optimal model-based control with limited communication." 19th IFAC World (2014).

[16] Çela, Arben, et al. "Optimal Integrated Control and On-line Scheduling of Resource-Constrained Systems." Optimal Design of Distributed Control and Embedded Systems. Springer International Publishing, 2014. 81-107.

[17] Longo, Stefano, et al. Optimal and robust scheduling for networked control systems. CRC Press, 2013.

[18] Zhang, Lei, and Dimitrios Hristu-Varsakelis. "Communication and control co-design for networked control systems." Automatica 42.6 (2006): 953-958.

[19] Chen, Xi, et al. "DTS: Dynamic TDMA scheduling for networked control systems." Journal of Systems Architecture 60.2 (2014): 194-205.

[20] Farokhi, Farhad, and Karl H. Johansson. "Stochastic sensor scheduling for networked control systems." Automatic Control, IEEE Transactions on 59.5 (2014): 1147-1162.

[21] Liu, Kun, and Emilia Fridman. "Discrete-time network-based control under scheduling and actuator constraints." International Journal of Robust and Nonlinear Control (2014).

[22] Gaid, Mohamed El Mongi Ben, and Arben Çela. "Trading quantization precision for update rates for systems with limited communication in the uplink channel." Automatica 46.7 (2010): 1210-1214.

[23] Donkers, M. C. F., Paulo Tabuada, and W. P. M. H. Heemels. "Minimum attention control for linear systems." Discrete Event Dynamic Systems 24.2 (2014): 199-218.

[24] Simon, Daniel, Ye-Qiong Song, and Christophe Aubrun. Co-design approaches to dependable networked control systems. John Wiley \& Sons, 2013.

[25] Mostofi, Yasamin. "Communication-aware motion planning in fading environments." Robotics and Automation, 2008. ICRA 2008. IEEE International Conference on. IEEE, 2008.

[26] Tang, Poi Loon, and Clarence W. De Silva. "Compensation for transmission delays in an ethernetbased control network using variable-horizon predictive control." Control Systems Technology, IEEE Transactions on 14.4 (2006): 707-718.

[27] Srinivasagupta, Deepak, Heinz Schättler, and Babu Joseph. "Time-stamped model predictive control: an algorithm for control of processes with random delays." Computers \& Chemical Engineering 28.8 (2004): 1337-1346.

[28] Pawlowski, Andrzej, et al. "Generalized predictive control with actuator dead band for event-based approaches." Industrial Informatics, IEEE Transactions on 10.1 (2014): 523-537.

[29] Rehbinder, Henrik, and Martin Sanfridson. "Scheduling of a limited communication channel for optimal control." Automatica 40.3 (2004): 491-500.

[30] Nilsson, Johan. Real-time control systems with delays. Diss. Lund institute of Technology, 1998.

[31] Yang, Shuang-Hua. Internet-based Control Systems: Design and Applications. Springer Science \& Business Media, 2011.

[32] Hristu-Varsakelis, Dimitris. "Short-period communication and the role of zero-order holding in networked control systems." Automatic Control, IEEE Transactions on 53.5 (2008): 1285-1290.

[33] Suzuki, Tatsuo, et al. "Controllability and stabilizability of a networked control system with periodic communication constraints." Systems \& Control Letters 60.12 (2011): 977-984.

[34] Longo, Stefano, Guido Herrmann, and Phil Barber. "Controllability, observability in networked control." Robust Control Design. Vol. 6. No. 1. 2009. 
[35] Tang, Xian-Ming, and Jin-Shou Yu. "Feedback scheduling of model-based networked control systems with flexible workload." International Journal of Automation and Computing 5.4 (2008): 389-394.

[36] Camacho, Eduardo F., and Carlos Bordons Alba. Model predictive control. Springer Science \& Business Media, 2013.

[37] Mayne, David Q., et al. "Constrained model predictive control: Stability and optimality." Automatica 36.6 (2000): 789-814.

[38] Löfberg, Johan. "YALMIP: A toolbox for modeling and optimization in MATLAB." Computer Aided Control Systems Design, 2004 IEEE International Symposium on. IEEE, 2004.

[39] Dua, Vivek, Nikolaos A. Bozinis, and Efstratios N. Pistikopoulos. "A multiparametric programming approach for mixed-integer quadratic engineering problems." Computers \& Chemical Engineering 26.4 (2002): 715-733.

[40] Axehill, Daniel. "Integer quadratic programming for control and communication." (2008).

[41] G. C. Goodwin, M. M. Seron, and J. A. De Doná." Constrained Control and Estimation - An Optimisation Approach", Springer Verlag, 2005.

[42] Bliek1ú, Christian, Pierre Bonami, and Andrea Lodi. "Solving Mixed-Integer Quadratic Programming problems with IBM-CPLEX: a progress report." (2014).

[43] Boyd, Stephen, and Lieven Vandenberghe. Convex optimization. Cambridge university press, 2004.

[44] He, Jian-qiang, Huan-chun Zhang, and Ya-zhi Jing. "An integrated control and scheduling optimization method of networked control systems." Journal of Electronic Science and Technology of China 2.2 (2004): 56-59.

[45] Walsh, Gregory C., Hong Ye, and Linda G. Bushnell. "Stability analysis of networked control systems." Control Systems Technology, IEEE Transactions on 10.3 (2002): 438-446.

[46] Branicky, Michael S., Stephen M. Phillips, and Wei Zhang. "Scheduling and feedback co-design for networked control systems." Decision and Control, 2002, Proceedings of the 41st IEEE Conference on. Vol. 2. IEEE, 2002.

[47] Park, Hong Seong, et al. "A scheduling method for network-based control systems." Control Systems Technology, IEEE Transactions on 10.3 (2002): 318-330.

[48] Zhao, Y. B., G. P. Liu, and D. Rees. "Integrated predictive control and scheduling co-design for networked control systems." IET Control Theory \& Applications 2.1 (2008): 7-15.

[49] Hristu-Varsakelis, Dmitris, and Panganamala R. Kumar. "Interrupt-based feedback control over a shared communication medium." Decision and Control, 2002, Proceedings of the 41st IEEE Conference on. Vol. 3. IEEE, 2002.

[50] Heemels, W. P. M. H., J. H. Sandee, and P. P. J. Van Den Bosch. "Analysis of event-driven controllers for linear systems." International journal of control 81.4 (2008): 571-590.

[51] Molin, Adam, and Sandra Hirche. "On LQG joint optimal scheduling and control under communication constraints." Decision and Control, 2009 held jointly with the 2009 28th Chinese Control Conference. CDC/CCC 2009. Proceedings of the 48th IEEE Conference on. IEEE, 2009.

\section{AUTHORS}

M.M. Share Pasand was born in 1984, received his BSc. and MSc. degrees in control engineering from Sharif and KNT Universities. He is a research faculty at Standard Research Institute. His research interest includes networked control and linear systems theory

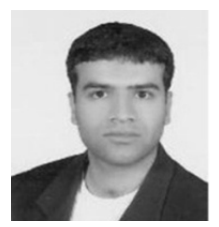

